\title{
Rise in Chronic Defibrillation Energy Requirements Necessitating Implantable Defibrillator Lead System Revision
}

\author{
EMILE G. DAOUD, K. CHING MAN, FRED MORADY, \\ and S. ADAM STRICKBERGER \\ From the Division of Cardiology, Department of Internal Medicine, \\ University of Michigan Hospitals, Ann Arbor, Michigan
}

\begin{abstract}
DAOUD, E.G., ET AL.: Rise in Chronic Defibrillation Energy Requirements Necessitating Implantable Defibrillator Lead System Revision. The chronic defibrillation energy requirement (DER) is believed to remain clinically stable in patients with defibrillators. Six patients (two with an epicardial and four with a nonthoracotomy system) were identified with a rise in their chronic DER, which eliminated a 10-J safety margin, thus necessitating a defibrillator lead system revision. The mean increase in DER was $14.7 \pm 4 \mathrm{~J}$ and was discovered at a mean of $16.0 \pm 18$ months (range 2-41) following implantation. Management included placement of a defibrillator with a biphasic waveform, placement of an additional defibrillation electrode, or both. At 2 months following revision of the defibrillation system, a 10-J DER safety margin was present in each patient. In some patients, there is a progressive increase in thè chronic DER with elimination of a 10-J safety margin necessitating revision of the defibrillation system. Routine reevaluation of the chronic DER, therefore, is necessary to identify these patients. (PACE 1997; 20[Pt. I]:714-719)
\end{abstract}

defibrillation energy, implantable defibrillator

\section{Introduction}

Either an epicardial or a nonthoracotomy system (NTL) can be used in combination with an implantable cardioverter defibrillator (ICD). The chronic defibrillation energy requirement (DER) with an epicardial lead system is thought to remain stable. ${ }^{1}$ However, with an NTL system, the DER may rise significantly during the first 2 months after implantation, although an adequate defibrillation safety margin has persisted. ${ }^{2}$ The purpose of this report is to describe six patients, four with an NTL system and two with an epicardial lead system, who had a rise in their DER 2-41 months after ICD implantation resulting in loss of an adequate defibrillation safety margin and requiring revision of the lead system.

Address for reprints: S. Adam Strickberger, M.D., Division of Cardiology, B1-F245, University of Michigan Hospitals, 1500 East Medical Center Drive, Ann Arbor, MI 48109-0022. Fax: (313) 936-7641.

Received August 29, 1995; revision November 27, 1995; accepted January 5, 1996.

\section{Methods}

Between November 1992 and January 1994, 6 of 162 patients ( $4 \%$ ) were prospectively identified to have a rise in the chronic DER that eliminated a 10-J defibrillation safety margin. In these patients, revision of the defibrillating lead system was performed.

\section{Clinical Characteristics}

The mean patient age was $64 \pm 10$ years (range 52-78); five patients were men. Five patients had an ischemic cardiomyopathy, and one patient had a nonischemic cardiomyopathy. The mean left ventricular ejection fraction was 0.22 . Four patients presented with cardiac arrest, one with syncope and one with sustained monomorphic ventricular tachycardia (VT). Five patients had inducible monomorphic VT at baseline programmed ventricular stimulation. The tachycardias were not suppressed with amiodarone therapy in two patients or sotalol therapy in two patients. Four patients had transvenous lead systems and two patients had epicardial lead systems. All patients had defibrillators that delivered monophasic shocks. 


\section{Determination of Acute DER}

The DER was determined either with successive successful shocks or with a step-down protocol until failure. For the two patients with epicardial lead systems, successive shocks, three shocks at $25 \mathrm{~J}$ or two shocks at $20 \mathrm{~J}$, were required. For the patients with NTL systems, the DER was determined by the step-down testing protocol. The acute DER was defined to be the lowest energy that successfully defibrillated ventricular fibrillation (VF) to sinus rhythm. Routinely, testing began at $25 \mathrm{~J}$, then $20,15,10,5$, and finally $1 \mathrm{~J}$. If $25 \mathrm{~J}$ failed, the electrode polarity was reversed and the step-down protocol was retested, beginning at $25 \mathrm{~J}$. If $25 \mathrm{~J}$ failed with reverse polarity, the DER was defined as $>25 \mathrm{~J}$ and a sub- cutaneous patch was added. An adequate de- fibrillation safety margin was defined as a DER at least $10 \mathrm{~J}$ less than the maximum ICD output.

\section{Initial ICD System and Acute DER Results (Table I)}

In each patient, the initially implanted ICD generator delivered shocks with a monophasic waveform. For the two patients with an epicardial lead system, defibrillation in one patient was between a left ventricle epicardial patch and a transvenous spring coil lead positioned in the superior vena cava. In the second patient, defibrillation was between two large epicardial patches.

For the four patients with NTL systems, one patient required a transvenous lead alone, one patient required a transvenous lead with a subcutaneous patch electrode, and two patients required two transvenous electrodes and a subcutaneous patch electrode. Each lead was placed through a left subclavian vein and one lead was positioned in the right ventricular apex. In the patients with two transvenous leads, the second lead was positioned at the superior vena cava-left brachycephalic vein junction in one patient, and in the coronary sinus in the other patient. The subcutaneous patch electrodes were placed through an incision in the anterior axillary line extending over the apex of the left ventricle. The patch was placed posteriorly extending beyond the posterior axillary line.

\section{Postimplant Care}

All patients had predischarge testing of the ICD. Testing included induction of VF and defibrillation at the maximum programmable output of the ICD generator. In patients with an NTL system, initial outpatient follow-up was in the electrophysiology laboratory 2 months following implantation. VF was induced and proper ICD sensing and defibrillation were tested at the programmed output of the generator. Testing was complete if the initial ICD shock successful defibrillated VF to sinus rhythm. Subsequent follow-up was at 2- to 3-month intervals in the outpatient ICD clinic.

\section{ICD Replacement for Battery End-of-Life}

When the battery charge was low, the generator was electively replaced in the electrophysiology laboratory with the patient under sedation with intravenous midazolam and fentanyl. The ICD pocket was opened and the leads were removed from the generator. Capture threshold, sensitivity, and impedance were measured for the ventricular sensing leads. Ventricular fibrillation was then induced with alternating current through the ventricular sensing leads. Defibrillation testing, as described above, was completed to confirm a 10-J DER safety margin.

\section{Evaluation of the Lead System}

Once the chronic DER was documented to have risen and eliminated a $10-\mathrm{J}$ safety margin, noninvasive techniques were used to investigate potential etiologies for the rise in DER. The position and integrity of the lead system was evaluated with chest roentgenograms. Ventricular capture threshold and sensitivity were determined for the sensing lead. The shocking lead impedance was also noted for the defibrillating electrodes, either noninvasively, if available, or at the time of revision of the defibrillation system. If these values were appropriate, a surface echocardiogram was performed to assess a change in left ventricular anatomy or function.

\section{Statistical Analysis}

Continuous variables are expressed as mean \pm 1 SD. Differences between continuous variables were tested by Student's $t$-test. $\mathrm{P}<0.05$ was considered significant. 
Table I.

Summary of Patient Management

\begin{tabular}{|c|c|c|c|c|c|c|c|}
\hline Patient & Initial Lead System & $\begin{array}{l}\text { Acute } \\
\text { DER }\end{array}$ & $\begin{array}{l}\text { Time From } \\
\text { Implantation to } \\
\text { Detection of } \\
\text { Rise in } \\
\text { Chronic DER } \\
\text { (months) }\end{array}$ & $\begin{array}{l}\text { Mode of Identifying Rise } \\
\text { in Chronic DER }\end{array}$ & $\begin{array}{l}\text { Chronic } \\
\text { DER }\end{array}$ & $\begin{array}{l}\text { Intervention to } \\
\text { Achieve 10-J } \\
\text { Safety Margin }\end{array}$ & $\begin{array}{l}\text { 2-Month } \\
\text { Follow-Up } \\
\text { DER }\end{array}$ \\
\hline 1 & $\begin{array}{l}1 \text { Epicardial patch with an } \\
\text { SVC electrode }+ \\
\text { monophasic ICD }\end{array}$ & $<15 \mathrm{~J}$ & 41 & ICD generator replacement & $>30 \mathrm{~J}$ & Biphasic ICD & $15 \mathrm{~J}$ \\
\hline 2 & $\begin{array}{l}2 \text { Epicardial patches }+ \\
\text { monophasic ICD }\end{array}$ & $<20 \mathrm{~J}$ & 36 & ICD generator replacement & $30 \mathrm{~J}$ & $\begin{array}{l}\text { SVC electrode }+ \\
\text { biphasic ICD }\end{array}$ & $10 \mathrm{~J}$ \\
\hline 3 & $\begin{array}{l}2 \text { NTLs with an SQ patch }+ \\
\text { monophasic ICD }\end{array}$ & $18 \mathrm{~J}$ & 13 & Evaluation of 1 year DER & $\geq 34 \mathrm{~J}$ & Patient refused & $\begin{array}{l}\text { Not } \\
\text { available }\end{array}$ \\
\hline 4 & $\begin{array}{l}\text { NTL with an SQ patch }+ \\
\text { monophasic ICD }\end{array}$ & $25 \mathrm{~J}$ & 2 & Routine reevaluation of ICD & $>34 \mathrm{~J}$ & $\begin{array}{l}\text { Removal of SQ } \\
\text { patch; } \\
\text { Placement of } 1 \\
\text { epicardial } \\
\text { patch }\end{array}$ & $<20 \mathrm{~J}$ \\
\hline 5 & NTL + monophasic ICD & $15 \mathrm{~J}$ & 2 & Routine reevaluation of ICD & $>34 \mathrm{~J}$ & $\begin{array}{l}2 \text { Epicardial } \\
\text { patches }\end{array}$ & $<24 \mathrm{~J}$ \\
\hline 6 & $\begin{array}{l}2 \text { NTLs with an SQ patch }+ \\
\text { monophasic ICD }\end{array}$ & $18 \mathrm{~J}$ & 2 & $\begin{array}{l}\text { Evaluation for pacemaker- } \\
\text { ICD interaction }\end{array}$ & $>34 \mathrm{~J}$ & Biphasic ICD & $27 \mathrm{~J}$ \\
\hline
\end{tabular}

$\mathrm{DER}=$ defibrillation energy requirement; $\mathrm{ICD}=$ implantable cardioverter defibrillator; $\mathrm{NTL}=$ nonthoracotomy lead; $\mathrm{SQ}=$ subcutaneous; $\mathrm{SVC}=$ superior vena cava.

\section{Results}

The acute DER was determined with successive successful shocks for the two patients with epicardial lead systems. The DER was $<15 \mathrm{~J}$ in the patient with the single left ventricular patch and $<20 \mathrm{~J}$ for the patient receiving two large patches. The acute DER for the transvenous lead systems was determined by the step-down protocol and was $15 \mathrm{~J}$ with a lead-alone configuration, $25 \mathrm{~J}$ with a lead and a subcutaneous patch, and $18 \mathrm{~J}$ in the two patients requiring two leads and a subcutaneous patch electrode. Predischarge testing of the ICD system demonstrated proper sensing and defibrillation of VF with a single ICD shock for all patients.

\section{Rise in DER at 2 Months (Table I)}

Three patients were identified to have a rise in their DER beyond a 10-J safety margin at 2 months following ICD implantation. No patients had required ICD therapies since implantation; in one patient, beta-blockade therapy was initiated. In each patient, $34 \mathrm{~J}$ failed to defibrillate VF. The integrity of each NTL was evaluated noninvasively and was intact. Management of the rise in DER was focused on altering the defibrillation configuration. In one patient, the subcutaneous patch electrode was removed and a single large epicardial patch was implanted. The acute DER was $10 \mathrm{~J}$. In a second patient, the addition of a subcutaneous defibrillating patch, reversing the shocking polarity, and testing multiple defibrillation configurations all failed to lower the DER and two epicardial patches were placed. The acute DER was $20 \mathrm{~J}$. In the final patient, 2 months after initial ICD implantation, a pacemaker was implanted. When evaluating for pacemaker-ICD interaction, VF failed to convert to sinus rhythm with a 34-J shock. Since multiple defibrillating configurations and polarities were assessed during the initial ICD implantation, the implanting shocking configuration was assumed to be optimal, and the ICD was replaced with one that de- 
livered a biphasic waveform with a maximum output of $37 \mathrm{~J}$. The acute DER was $27 \mathrm{~J}$.

\section{Rise in DER Beyond 2 Months (Table I)}

In the two patients with epicardial lead systems, the rise in DER was identified at the time of elective replacement of the ICD generator, $38 \pm 4$ months after initial epicardial ICD system implantation. Each patient had appropriate ICD shock therapy with their original ICD system. One patient had one episode of syncope associated with three sequential shocks. In both patients, the integrity of the leads was intact and a transthoracic echocardiogram revealed no significant changes in left ventricular structure and function compared to the time of initial ICD implantation. In one patient, the chronic DER rose to $>30 \mathrm{~J}$. Despite reversing the defibrillation polarity, ${ }^{3,4}$ the DER was unchanged and an ICD with a biphasic waveform was implanted. The DER was then $15 \mathrm{~J}$.

For the second patient with an epicardial lead system, the chronic DER rose to $34 \mathrm{~J}$. A superior vena cava electrode was placed through the left subclavian vein, and the DER was $25 \mathrm{~J}$. At predischarge testing, VF was cardioverted to sinus rhythm by a 34-J shock. Three months later, during evaluation for pacemaker-ICD interaction, the DER was found to be $30 \mathrm{~J}$. Reversing ICD electrode polarity or altering the electrode configuration were unsuccessful in achieving a 10-J DER safety margin. An ICD with a biphasic waveform was implanted and the step-down DER was $10 \mathrm{~J}$. In the remaining patient, the rise in the chronic DER was identified at 1-year evaluation of the DER. The patient had no ICD therapies since implantation and his chronic DER rose from $18 \mathrm{~J}$ to $>34 \mathrm{~J}$. The defibrillation system included two transvenous leads and a subcutaneous patch. We anticipated opening the ICD pocket and testing reverse polarity, and if this was unsuccessful in lowering the DER, a biphasic waveform device was to be implanted. The patient, however, refused any further procedures and left the hospital against medical advice. The patient has been followed for 5 months and has not received any ICD therapies.

\section{Defibrillation Impedance}

The defibrillation impedance at the time of initial ICD implantation was $45 \pm 7.3 \Omega$. The impedance at the time of lead revision was $49 \pm$ $6.8 \Omega(\mathrm{P}=0.33)$.

\section{Two-Month Follow-Up}

Repeat evaluation of the DER was completed 2 months after revision of the ICD system. The DER was at least $10 \mathrm{~J}$ less than the maximum programmable output of the ICD in each patient.

\section{Discussion}

These six cases document that a progressive increase in DER may occur more than 6 months after ICD implantation, both with NTL and epicardial lead systems. The increased energy requirement may eliminate a 10-J defibrillation safety margin present at the time of the initial implant and necessitate a change in either the defibrillation configuration or the shock waveform.

In 5 of the 6 patients, a 10-J DER safety margin was achieved by sequentially testing four methods: (1) reversal of the defibrillation polarity ${ }^{3,4}$; (2) placement of a defibrillating electrode or subcutaneous or epicardial patch; (3) placement of an ICD that delivers a biphasic waveform ${ }^{5,6}$; or (4) a combination of these three methods. This stepwise approach is based on achieving an adequate DER by the least invasive and perhaps the most cost-effective revision of the ICD system. Placement of an electrode or patch was successful in two patients, changing to a biphasic waveform ICD was successful in two patients, and placement of an electrode and changing to a biphasic waveform ICD was successful in one patient.

The clinical significance of a rise in the DER and loss of a 10-J safety margin is unknown. In five patients, the rise in the DER was detected during routine ICD evaluation. One patient in this series presented with syncope and required multiple shocks. In this patient, the requirement for multiple shocks implies that absence of a 10-J safety margin may hold clinical significance. In the absence of shock therapy, as in the other five patients, the clinical significance of this finding is uncertain.

\section{Previous Reports}

In other studies, a significant rise in DER was noted to occur during the first 2 months after implantation of an NTL system, ${ }^{2}$ but not after im- 
plantation of an epicardial lead system. ${ }^{1}$ The observed DER elevation after NTL system implantation did not exceed adequate defibrillation energy safety margins, and revision of the system was not required in these studies. However, other investigators have reported that a rise in the chronic DER can occur and that ICD system revision may be necessary. ${ }^{7-9}$

\section{Mechanisms of Increase in Chronic DER}

The mean increase in chronic DER was $\geq 14.7$ $\pm 4 \mathrm{~J}$. The etiology of this increase is unclear. There are four potential mechanisms. At any given energy, successful defibrillation of VF is based on a probability curve. ${ }^{10}$ It is possible that the rise in DER determined at follow-up testing represents a statistical phenomenon and repeated testing at the same energy would reveal greater defibrillation success. This theory seems unlikely, however, since the follow-up DER was determined by the step-down protocol. This method of DER determination correlates with approximately $70 \%$ of success in animal models. ${ }^{11}$

A loss of lead or patch integrity, position, or contact with the myocardium is a second mechanism for a rise in chronic DER. Capture threshold, impedance, sensitivity, and, if possible, shocking impedance were determined. Progressive scarring and fibrosis around the defibrillation coils or patches could raise the shocking impedance and, for epicardial patches, effusions have been noted to develop between the patches and the myocardium, which also raises the impedance. ${ }^{12}$ An increase in the shocking impedance will decrease the delivered defibrillation current and possibly raise the DER. In this study, there was no significant change in the impedance measured at implantation versus that at the time of revision. Additionally, follow-up chest roentgenograms were compared to predischarge films to evaluate for lead dislodgment or fracture or crinkling of epicardial or subcutaneous patches. Although an evaluation for epicardial patch effusion was not completed for our patients, the other investigated parameters did not reveal a lead or patch complication.
Another possible mechanism for a rise in the chronic DER is an interval change in the myocardial substrate. An intercedent event, such as a myocardial infarction, increase in heart size, or decrease in ejection fraction, may require greater defibrillation energy. No patient had an intercedent myocardial infarction and repeat transthoracic echocardiograms were unchanged. This was not noted in these patients.

Finally, alterations in autonomic tone or drug therapy may affect DER. ${ }^{13,14}$ In one patient blockade therapy was initiated. A recent study suggests that $\beta$-antagonists do not increase the DER ${ }^{15}$; however, other antiarrhythmic agents have been shown to increase the DER. ${ }^{16}$

\section{Limitations}

This study is limited by the small sample size. However, there have been no previous reports demonstrating the loss of an adequate defibrillation safety margin resulting in lead system revision. An additional limitation is that each patient initially had a defibrillator with a monophasic waveform; thus, these observations may not apply to patients with a biphasic waveform device.

\section{Clinical Implications}

These patients highlight the limited data that exist about the chronic DER and, hence, the importance of routine reevaluation of the chronic DER 2 months after initial implantation of an ICD with an NTL system, and at the time of ICD replacement for both epicardial and NTL systems. Three of the six patients in this report were identified during a standard 2-month follow-up of the chronic DER, and two patients were identified at the time of ICD generator change approximately 3 years following the initial implant date. If the lead system is demonstrated to be intact, management of these patients should be directed at obtaining a 10-J DER safety margin by revising either the defibrillation lead configuration or the ICD defibrillation waveform. 


\section{RISE IN CHRONIC DER}

\section{References}

1. Wetherbee JN, Chapman PD, Troup PJ, et al. Longterm internal cardiac defibrillation threshold stability. PACE 1989; 12:443-450.

2. Venditti FJ, Martin DT, Vassolas G, et al. Rise in chronic defibrillation thresholds in nonthoracotomy implantable defibrillator. Circulation 1994; 89:216-223.

3. Strickberger SA, Hummel JD, Horwood L, et al The effect of polarity on defibrillation threshold using a non-thoracotomy lead system. J Am Coll Cardiol 1994; 24:1069-1072.

4. Bardy GH, Ivey TD, Allen MD, et al. Evaluation of electrode polarity on defibrillation efficacy. Am J Cardiol 1989; 60:618-624.

5. Swartz JF, Fletcher RD, Karasik PE. Optimization of biphasic waveforms for human nonthoracotomy defibrillation. Circulation 1993; 88:2646-2654.

6. Neuzner J, Pitschner HF, Huth C, et al. Effect of biphasic waveform pulse on endocardial defibrillation efficacy in humans. PACE 1994; 17: 207-212.

7. Nunain SO, Roelke M, Trouton T, et al. Limitations and late complications of third-generation automatic cardioverter-defibrillators. Circulation 1995; 91:2204-2213.

8. Schwartzman D, Nallamothu N, Callans DJ, et al Postoperative lead-related complications in patients with nonthoracotomy defibrillation lead systems. J Am Coll Cardiol 1995; 26: 776-786.

9. Natale A, Sra J, Axtell K, et al. Preliminary experience with a hybrid nonthoracotomy defibrillating system that includes a biphasic device: Comparison with a standard monophasic device using the same lead system. J Am Coll Cardiol 1994; 24 : $406-412$.

10. Singer I, Lang D. Defibrillation threshold: Clinical utility and therapeutic implications. PACE 1992; 15:932-949.

11. Lang DJ, KenKnight BH: Implant support devices. In I Singer (ed.): Implantable Cardioverter-Defibrillator. Armonk, NY, Futura Publishing Co., Inc., 1994, p. 223.

12. Goodman LR, Hossein AG, Troup PI, et al. Complications of automatic implantable cardioverter defibrillators: Radiographic, CT, and echocardiographic evaluation. Radiology 1989; 170:447-452.

13. Sousa J, Kou W, Calkins $H$, et al. Effect of epinephrine on the efficacy of the internal cardioverter-defibrillator. Am J Cardiol 1992; 69: 509-512.

14. Rigden LB, Mitrani RD, Rardon DP, et al. Diffuse cardiac sympathetic dysfunction follows implantable defibrillator shocks delivered through epicardial patch electrodes. (abstract) J Am Coll Cardiol 1995; 25:85A.

15. Lemmer JH, Smith BG, Mower MM, et al. Chronic atenolol and defibrillation energy requirements. Circulation 1993; 88:I-113.

16. Echt DS, Gremillion ST, Lee JT, et al. Effects of procainamide and lidocaine on defibrillation energy requirements in patients receiving implantable cardioverter defibrillator devices. J Cardiovasc Electrophysiol 1994; 5:752-760. 
This document is a scanned copy of a printed document. No warranty is given about the accuracy of the copy. Users should refer to the original published version of the material. 